\title{
Comparative Study of Surgical Approaches for Distractive Flexion Injuries of Sub-Axial Cervical Spine
}

\author{
Hatem M. Al Samouly, Ahmed M. Taha \\ Departments of Neurosurgery, Damietta Hospital, Al-Azhar University, Cairo, Egypt \\ Email: ahmdth231@gmail.com
}

How to cite this paper: Al Samouly, H.M. and Taha, A.M. (2018) Comparative Study of Surgical Approaches for Distractive Flexion Injuries of Sub-Axial Cervical Spine. Open Journal of Modern Neurosurgery, 8, 342-351.

https://doi.org/10.4236/ojmn.2018.83029

Received: May 11, 2018

Accepted: July 28, 2018

Published: July 31, 2018

Copyright $\odot 2018$ by authors and Scientific Research Publishing Inc. This work is licensed under the Creative Commons Attribution International License (CC BY 4.0).

http://creativecommons.org/licenses/by/4.0/

\section{(c) (†) Open Access}

\begin{abstract}
Distractive flexion injuries (DFI) of subaxial cervical spine are common after motor car accident or falling from height. The ideal surgical approaches to DFI are still unclear. A retrospective comparative study of surgical approaches for DFI of sub-axial cervical spine involved 60 patients throughout the years 2014 to 2016 at Al-Azhar University Hospitals. All patients were undergoing initial routine resuscitative measures, full general and neurological examinations. Neurologic function was assessed according to modified Frankel's grading. All patients were received cervical plain antero-posterior, lateral and oblique X-ray, CT with 3D and MRI of cervical spine. Severity of DFI injury was assessed according to Allen and Ferguson's classification. The most common level involved was C5-6 and most common grade was grade 3 . The patients operated through anterior approach were 36 patients and through the posterior approach were 24 patients. Restoring cervical alignment was achieved in 29 patients (80.56\%). The mean time of bone fusion was 5.454 months in anterior approach while it was 9.876 months in posterior approach. The extend of bone fusion was good in 30 patients (83.33\%) after anterior approach and in 8 patients (33.33\%) after posterior approach, while poor fusion was observed in 6 patients $(16.67 \%)$ after anterior approach and 16 patients (66.67\%) after posterior approach. We can conclude that anterior cervical approach is better in DFI stage 3 and 4 where there are associated ruptured intervertebral disc. Posterior approach is better in DFI stage 1 and 2 with or with presence of posterior compressing lesion.
\end{abstract}

\section{Keywords}

Distractive Flexion Injuries, Sub-Axial Cervical Spine 


\section{Introduction}

Distractive flexion injuries (DFI) of subaxial cervical spine constitute $10 \%$ of all injuries to lower cervical spine. The main cause is road traffic injuries. This injury involves posterior soft tissue and not bone, so the healing with conservative treatment is poor. This injury is due to hyperflexion of cervical spine leading to disruption of interspinous, supraspinous ligaments, ligamentum flavum, facet capsule, the anterior longitudinal ligament (ALL), posterior longitudinal ligament (PLL), and disc. Although the disruption of the posterior ligaments is severe, all three columns are usually affected [1]. DFI of subaxial cervical spine are graded along a spectrum that includes subluxation, perched facts, unilateral dislocation, bilateral facet dislocations and fracture dislocation [2]. MRI allows visualization of disruptions of posterior and anterior longitudinal ligaments and facet capsules. The ability of MRI to detect a canal occupying herniated disc before or after closed skeletal cervical traction results in the potential for variability in surgical approach selection [3]. The reduction and restoration of cervical alignment is necessary. The surgical approach is variable depending on severity of DFI, spinal cord compression, presence of a traumatic intervertebral disc herniation, neurologic status, and the technical familiarity of the individual surgeon [4].

\section{Patients \& Methods}

A retrospective comparative study of 60 consecutive patients with DFI of subaxial cervical spine, was operated over the period 2014 to 2016. All patients were undergoing initial routine resuscitative measures, full general and neurological examinations. Assessing the neurologic function according to modified Frankel's grading: Grade A: Complete quadriplegia, Grade B: Incomplete quadriplegia with useless motor function, Grade C: Incomplete quadriplegia with useful motor function, Grade D: Root sign or symptom and Grade E: Intact motor and sensory function. All patients were received cervical plain antero-posterior, lateral and oblique X-ray, CT with 3D and MRI of cervical spine. Severity of DFI injury was assessed according to Allen and Ferguson's classification. It includes:

DFI stage 1: Flexion sprain and fact joint subluxation with divergence of the spinous process.

DFI stage 2: Unilateral facet joint dislocation.

DFI stage 3: Bilateral facet joint dislocation, with translation is less than $50 \%$.

DFI stage 4: Floating vertebrae, with translation is more than $50 \%$.

Distractive flexion injuries associated with other cervical spine injuries or more than one level involved were excluded from the study. Closed skull traction was applied after MRI cervical spine to to excludes traumatic disc herniation. Surgery was started within $8-72$ hours from the accident in all patients.

\section{Surgical procedures:}

Anterior approach: supine position, fibro-optic intubation, anterior cervical approach which includes discectomy, unlocking facet joint through controlled 
distraction and bone fusion using cervical cage with plate and screws.

Posterior approach: fibro-optic intubation, prone position, posterior cervical approach which includes unlocking facets through partial superior facetectomy of the involved fact and manipulation, fusion with bone graft and lateral mass fixation using screws and rods. All patients wore postoperatively hard cervical collar for 6 weeks. Compared and followed up of all patients clinically with periodic assessment of neurological recovery and plain radiographs at 3 months, 9 months and at 12 months. The fusion rate and alignment of cervical spine were assessed. Management of the operative complications in both approaches. Statistical analysis: Data was analyzed by Microsoft Office XP 2010 (excel) and Statistical Package for Social Science (SPSS) version 10. Parametric data was expressed as mean $\pm \mathrm{SD}$, and non-parametric data was expressed as number and percentage of the total.

\section{Results}

Sixty consecutive patients with DFI of sub-axial cervical spine were admitted and operated at Al-Azhar University Hospitals throughout the years 2014 to 2016. Males were $42(70 \%)$ and females were $18(30 \%)$ with the age ranged from 18 to 52 years ( $38.23 \pm 14.38$ years). The mechanism of injury was motorcar accident in 51 patients (85\%) and falling from height in 9 patients (15\%). The most common level involved was C5 - 6 (41.66\%). The radiological criteria in MRI, $\mathrm{CT}$ and plain X-ray cervical are used to classify the level involved. The most common DFI stage according to Allen and Ferguson's classification, was DFI stage 3, (24) patients (40\%) (Table 1).

The most common neurological grade according to to modified Frankel's grading scale was grade A, (20) patients (40\%) was most common grade presented among studied patients (Table 2).

The patients operated through anterior approach were 36 patients $(60 \%)$ including stage 1, ( 3 cases) stage 2, ( 3 cases) stage 3, (24 cases) and 4 ( 6 cases). The patients operated through posterior approach were 24 patients (40\%) including stage $1,(15$ cases) and stage 2 , (9 cases) (Table 3$)$.

Table 1. Levels and stages of DFI in the cervical spine among studied patients.

\begin{tabular}{cccccccccc}
\hline & \multicolumn{4}{c}{ Level } & \multicolumn{7}{c}{ Stage of DFI } \\
\cline { 2 - 10 } Level/stage & C3 - 4 & C4 - 5 & C5 - 6 & C6 - 7 & Stage 1 & Stage 2 & Stage 3 & Stage 4 \\
\hline $\begin{array}{c}\text { Number } \\
\%\end{array}$ & 6 & 21 & 25 & 8 & 18 & 12 & 24 & 6 & 60 \\
& $10.00 \%$ & $35.00 \%$ & $41.66 \%$ & $13.33 \%$ & $30.00 \%$ & $20.00 \%$ & $40.00 \%$ & $10.00 \%$ & $100 \%$
\end{tabular}

Table 2. Grade of neurological function among studied patients.

\begin{tabular}{ccccccc}
\hline \multirow{2}{*}{ Neurological function } & \multicolumn{6}{c}{ Grade of neurological function } \\
\cline { 2 - 7 } & Grade A & Grade B & Grade C & Grade D & Grade E & Total \\
\hline Number & 24 & 12 & 9 & 10 & 5 & 60 \\
$\%$ & 40.00 & 20.00 & 10.66 & 16.66 & 8.33 & $100 \%$ \\
\hline
\end{tabular}


Table 3. DFI stages and the approach used among studied patients.

\begin{tabular}{cccc}
\hline & Anterior approach & Posterior approach & Number \\
\hline DFI stage 1 & 3 & 15 & 18 \\
DFI stage 2 & 3 & 9 & 12 \\
DFI stage 3 & 24 & - & 24 \\
DFI stage 4 & 6 & - & 6 \\
Total & 36 & 24 & 60 \\
$\%$ & $60 \%$ & $40 \%$ & $100 \%$ \\
\hline
\end{tabular}

The association of cervical disc herniation with DFI was 32 cases (53.3\%) as follow: DFI stage (1) 3 cases, stage (2) 3 cases, stage (3) 20 cases and stage (4) 6 cases. Skull traction was applied in 44 patients (73.3\%). All looked facet which failed in closed reduction were successfully open reduced during surgery either through anterior or posterior approach.

The complications of anterior approach were, transient laryngeal nerve palsy 2 cases, (5.56\%), transient dysphasia 2 cases (5.56\%), carotid artery injury one case (2.78\%), dural tear one case (2.78\%), and cord contusion one case $(2.78 \%)$. The complications of posterior approach were, screw loosening 5 case $(20.83 \%)$ which was statistically significant, vertebral artery injury one case (4.17\%) (Table 4).

In anterior approach the neurological recovery was observed during the fellow up period in 13 patients (36\%), and in posterior approach was 14 patients (58.3\%) (Table 5).

The restore of cervical alignment (Cobb's angle) was achieved in 29 patients (80.56\%) operated through anterior approach and in 12 patients (50\%) through posterior approach (Table 6).

The mean time of bone fusion was 5.454 months in anterior approach while it was 9.876 months in posterior approach (Table 7).

\section{Case Presentation}

\subsection{Case 1}

Male patient 36 years old had road traffic accident, with modified Frankel's grade $\mathrm{C}$ and DFI stage 3. Skull traction was applied to restore of cervical alignment. Rapid anterior cervical approach, discectomy, unlocking of the facets, fusion with cervical cage and fixation with plate and screws (Figure 1).

\subsection{Case 2}

Female patients 25 years old was felled from height with modified Frankel's grade D and DFI stage 2. Closed skull traction was failed. Posterior cervical approach, unlocking of the facet, bone fusion and lateral mass fixation with rods and screws (Figure 2). 
Table 4. Showed complications of both approaches.

\begin{tabular}{cccccc}
\hline \multirow{2}{*}{ Complications } & \multicolumn{2}{c}{ Anterior } & \multicolumn{3}{c}{ Posterior } \\
\cline { 2 - 6 } & Number & $\%$ & Number & $\%$ & P value \\
\hline Transient Laryngeal nerve palsy & 2 & 5.56 & 0 & 0.00 & 0.01 \\
Transient dysphasia & 2 & 5.56 & 0 & 0.00 & 0.01 \\
Carotid artery injury & 1 & 2.78 & 0 & 0.00 & 0.01 \\
Dural tear & 1 & 2.78 & 0 & 0.00 & 0.01 \\
Cord contusion & 1 & 2.78 & 0 & 0.00 & 0.01 \\
Screw loosening & 0 & 0.00 & 5 & 20.83 & 0.06 \\
Vertebral artery injury & 0 & 0.00 & 1 & 4.17 & 0.1 \\
\hline
\end{tabular}

*significant at $\mathrm{P}<0.05$.

Table 5. Showed neurological recovery from both approaches.

\begin{tabular}{ccccc}
\hline & \multicolumn{2}{c}{ Anterior Preoperative } & \multicolumn{2}{c}{ Posterior Preoperative } \\
\cline { 2 - 5 } & Number & $\%$ & Number & $\%$ \\
\hline Grade A & 18 & 38.89 & 6 & 75.00 \\
Grade B & 6 & 16.67 & 5 & 12.50 \\
Grade C & 6 & 13.89 & 4 & 8.33 \\
Grade D & 4 & 16.67 & 6 & 4.14 \\
Grade E & 2 & 13.89 & 3 & 0.00 \\
Total & 36 & $100 \%$ & 24 & $100 \%$ \\
& Anterior Postoperative & Posterior Postoperative \\
Grade A & Number & $\%$ & Number & $\%$ \\
Grade B & 13 & 33.33 & 5 & 50.00 \\
Grade C & 9 & 11.11 & 6 & 8.33 \\
Grade D & 5 & 11.11 & 1 & 16.67 \\
Grade E & 4 & 11.11 & 9 & 8.33 \\
Total & 5 & 33.33 & 3 & 16.67 \\
\hline
\end{tabular}

Table 6. Restore of cervical alignment in both approaches among studied patients.

\begin{tabular}{lccccc}
\hline & \multicolumn{2}{c}{ Anterior approach } & \multicolumn{2}{c}{ Posterior approach } & P value \\
\cline { 2 - 5 } & No. & $\%$ & No. & $\%$ & \\
\hline Restore of cervical alignment & 29 & 80.56 & 12 & 50.00 & $<0.01$ \\
\hline significant at $\mathrm{P}<0.05$. & & & & & \\
\hline sinn & & & &
\end{tabular}

Table 7. Time of bone fusion in both approaches among studied patients.

\begin{tabular}{cccccc}
\hline \multirow{2}{*}{$\begin{array}{c}\text { fusion time } \\
\text { (months) }\end{array}$} & \multicolumn{2}{c}{ Anterior Approach } & \multicolumn{2}{c}{ Posterior Approach } & \multirow{2}{*}{ P value } \\
\cline { 2 - 5 } & Mean & SD & Mean & SD & \\
\hline & 5.454 & 1.98 & 9.876 & 2.671 & $<0.01$ \\
\hline
\end{tabular}

*significant at $\mathrm{P}<0.05$. 

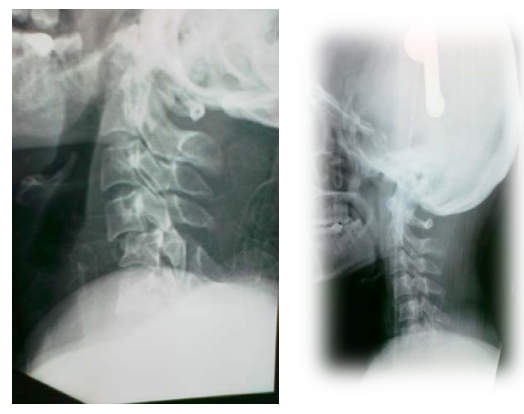

(a)

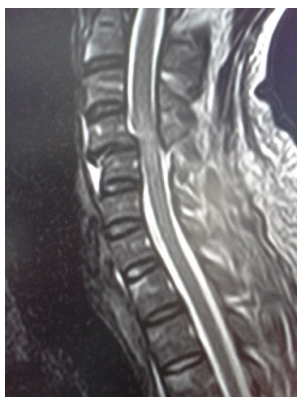

(b)

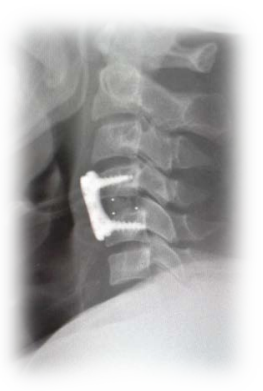

(c)

Figure 1. Showing case 1 presentation. (a) Pre-operative plain X-ray (b) pre-operative skull traction (c) MRI cervical spine (d) post-operative anterior cervical cage fusion and plate fixation.

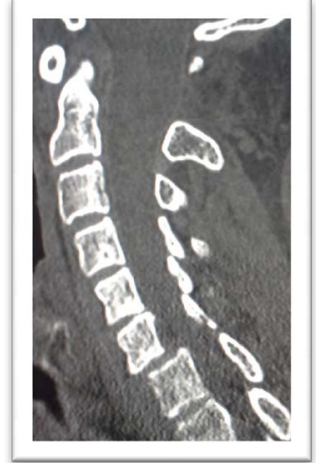

(a)

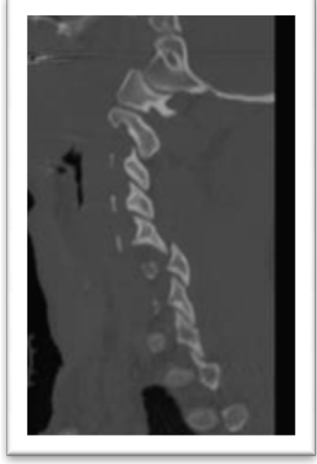

(b)

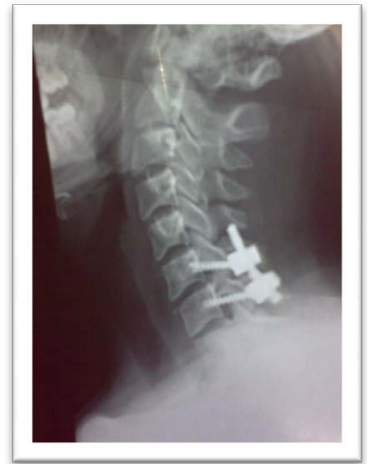

(c)

Figure 2. Showing case 2 presentation. (a) Pre-operative CT (b) pre-operative CT with bone window (c) post-operative plain X-ray lateral mass rode and screws fixation and fusion.

\section{Discussion}

The aim of treatment for distractive flexion injuries of sub-axial cervical spine is to decompress the neural canal, restore of cervical alignment by instrumentation and enhance fusion. This can be achieved through anterior or posterior and or combined approaches along with bone fusion [2]. As the instability in DFI is due to rupture of the posterior soft tissue, it has been the general idea to stabilize these injuries by a posterior approach and fixation using wires or plates. This viewpoint is also supported by biomechanical studies where anterior fixation proved insufficient, and the recommendation is to advocate a posterior instrumentation when treating DFI [5]. Failure to recognize the presence of three-column instability was believed to result in the failure of posterior tension band stabilization as means of gaining cervical spine stability [6]. Recommendation to perform both anterior and posterior fixation in DFI based on a clinical study and in a biomechanical investigation [7]. Most of the results in the literature show that with an anterior approach and proper application of a plate-bone construct can be reached in most instances. Complications have been advocated like dislodgement of implants, in most series reported below $5 \%$. There is obviously a 
risk to penetrate the disc space or the spinal canal [8]. Inappropriate plate application in osteoporotic bone may be another reason for implant failure [9]. This study is conducted on sixty consecutive patients which had DFI of the subaxial cervical spine of different stages and levels. patients operated through anterior approach were 36 and through posterior approach were 24 . it showed that anterior approach is better than posterior approach by restoring cervical alignment, enhance bone fusion and neurological recovery. This is supported by anterior approach allows the excision of offending disc herniation with subsequent enhance the neurological recovery [10]. Reconstruction of cervical spine with two columns stability is effective than one column in restoring alignment and enhance fusion [9]. Although the combined anterior and posterior fixation and fusion with DFI is superior to anterior fixation and fusion alone, but it can increase the morbidity of surgery due to longer operation, however there is no difference in the degree of neurological recovery. Recommendation of anterior fixation and fusion alone to be performed first followed by delayed posterior fixation and fusion if necessary at follow up period [10]. The use of anterior plate alone for DFI stage 1 and 2 but disqualify anterior plate fixation alone for DFI stage 3 and 4 with neurologic deficit [1]. This is coincided with our study which showed that anterior approach alone is sufficient to maintained the stability of injury. But we noticed an increased degree of neurological recovery in anterior approach rather than posterior approach. We operated 6 cases through anterior approach and 24 cases through posterior in DFI stage 1 and 2, and operate 24 cases through anterior approach in DFI stage 3 and 4 . The choice of posterior approaches in grade 1 and 2 based is the absence of disc herniation. Rapid skull traction and reduction should be performed for bilateral cervical locked facet dislocation, moreover posterior open reduction should be given if the closed reduction is fails, however, rapid skull traction and reduction is not appropriate for those with unilateral locked facet dislocation, and posterior open reduction is the first choice for these patients [11]. In our study rapid skull traction was applied to all patients after MRI cervical spine to determined the cervical disc herniation. If there is disc herniation with DFI stage 1 and 2, we operated the patient anteriorly and if there is no disc herniation we operated 3 patients anteriorly and 9 patients posteriorly. All patient with DFI stage 3 and 4 were operated posteriorly. Henriques et al., 2004 was not mentions the cervical disc herniation as a factor in decision of his approach [12]. The variations in surgical treatment of cervical facet dislocations and they recommended that, surgeons used more anterior approaches either alone or as the first stage in combined approach when a disc herniation was present regardless of neurologic status of the patients. When patients were neurologically intact, an anterior was more common than posterior approach even when a disc herniation was not present. Combined approaches were preferred for the treatment of bilateral facet dislocation. In our study all patients with cervical disc herniation regardless of grade of DFI were operated through anterior approach, and posterior approach in DFI grade 1 and 
2 in absence of cervical disc herniation [13]. Comparison the clinical and radiographic outcomes of patients with DFI of the subaxial cervical spine who had undergone a posterior procedure using cervical pedicle screw fixation with those who had undergone a combined anterior and posterior procedure suggested posterior procedures with cervical pedicles screws fixation for the management of cervical DFI [13]. In our study we clarified the grade of DFI, and we used the lateral mass fixation in stage land 2 without disc herniation. Anterior surgery may be contra-indicated in case of significant posterior lesions compromising the spinal cord, roots, in clinically relevant dural leaks, and in case of locked facet joints, which are unreducible by traction or even anterior open surgery, specifically, in case of delayed surgery. Furthermore, highly unstable injuries may need a combined anterior-posterior surgery or if an anterior stabilization may appear insufficient intraoperatively [10]. Posterior plating techniques have been reported to be better in controlling rotational instability, however, because of the weakness of the posterior structures, collapse of the disc space or kyphosis may develop in the late stage [14]. Posterior stabilization and fusion procedures led to unsuccessful results in five of the 11 patients (45\%), related either to late kyphosis because of disc collapse or the inability of midline stabilization procedures to control rotational instability [15]. Some authors have suggested that anterior stabilization techniques are biomechanically inferior to posterior stabilization techniques, specifically in the treatment of distractive flexion-type injuries [16]. In the prospective part of series, 18 patients were treated with anterior cervical decompression at a single space, fusion with autogenous tricortical iliac crest graft and stabilization with anterior cervical plate. In the follow-up of these cases (for at least 2 years), there was no evidence of inadequate fusion or nonunion, and no patient necessitated further surgery [17]. In the current study, the anterior approach succeeded in improving the neurological function in 13 (36\%) patients and the posterior approach in 14 (58.3\%) patients. Anterior decompression may allow for maximal neurological recovery in patients with incomplete neurological deficits. The neurological recovery may be attributed to rapid skull traction which gives the patients best chance of of recovery from compression of the spinal cord or nerve root or at least prevent progressive secondary spinal cord injury [16].

\section{Conclusion}

The surgical treatment of DF injuries of subaxial cervical spine remain controversial. Rapid realignment and decompressions of the sub axial cervical spine by skull traction are giving the patients best chance for neurological recovery and reduce secondary spinal cord injury. The optimal approach for distractive flexion injury to the cervical spine is the least invasive approach that provides the greatest benefit to risk ratio in terms of potential injuries to contiguous neurovascular structures and provides adequate stabilization. Anterior cervical approach is better in DFI stage 3 and 4 where there is associated ruptured inter- 
vertebral disc. Posterior cervical approach is better in DFI stage 1 and 2 without disc herniation or if there is a posterior compressing lesions. Realignment and Fusion is good in anterior than posterior approach. Anterior approach is more familiar and less predictable complications.

\section{References}

[1] Henriques, T., Olerud, C., Bergman, A. and Jonsson, H. (2004) Distractive Flexion Injury of the Subaxial Cervical Spine Treated with Anterior Plate Alone. Journal of Spinal Disorders \& Techniques, 17. https://doi.org/10.1097/00024720-200402000-00002

[2] Song, K.J. and Lee, K.B. (2008) Anterior versus Combined Anterior and Posterior Fixation/Fusion in the Treatment of Distractive-Flexion Injury in the Lower Cervical Spine. Journal of Clinical Neuroscience, 15, 36-42. https://doi.org/10.1016/j.jocn.2007.05.010

[3] Alexander, V.R., Luke, M.B.A., Mark, S.E., Adam, F.E. and Alan, H.S. (2001) Magnetic Resonance Imaging Analysis of Soft Tissue Disruption after Flexion-Distraction Injuries of the Subaxial Cervical Spine. Spine, 26, 1866-1872.

[4] Del Curto, D., Tamaoki, M.J., Martins, D.E., Puertas, E.B. and Belloti, J.C. (2014) Surgical Approaches for Cervical Spine Facet Dislocations in Adults. The Cochrane Database of Systematic Reviews, CD008129.

[5] Shapiro, S., Snyder, W., Kaufman, K. and Abel, T. (1999) Outcome of 51 Cases of Unilateral Locked Cervical Facets: Interspinous Braided Cable for Lateral Mass Plate Fusion Compared with Interspinous Wire and Facet Wiring with Iliac Crest. Journal of Neurosurgery, 91, 19-24. https://doi.org/10.3171/spi.1999.91.1.0019

[6] James, K. and Das, K. (2001) Posterior Fusion of the Subaxial Cervical Spine: Indications and Techniques. Neurosurgical Focus, 10, Article 7.

[7] Payer, M. (2005) Immediate Open Anterior Reduction and Anteroposterior Fixation/Fusion for Bilateral Cervical Locked Facets. Acta Neurochirurgica (Wien), 147, 509-513. https://doi.org/10.1007/s00701-004-0462-6

[8] Aebi, M., Zuber, K. and Marchesi, D. (1991) The Treatment of Cervical Spine Injuries by Anterior or Plating. Spine, 16, 38-45.

https://doi.org/10.1097/00007632-199103001-00008

[9] Reindl, R., Quellet, J. and Harvey, E. (2006) Anterior Reduction for Cervical Spine Dislocation. Spine, 12, 648-652. https://doi.org/10.1097/01.brs.0000202811.03476.a0

[10] Aebi, M. (2010) Surgical Treatment of Upper, Middle and Lower Cervical Injuries and Non-Unions by Anterior Procedures. European Spine Journal, 19, S33-S39. https://doi.org/10.1007/s00586-009-1120-8

[11] Yu, Z.S., Yue, J.J., Wei, F., Liu, Z.J., Chen, Z.Q. and Dang, G.T. (2007) Treatment of Cervical Dislocation with Locked Facets. Chinese Medical Journal (English Edition), 120, 216-218.

[12] Nasser, H., Lee, J.Y., Dvorak, M.F., Harrop, J.S., Dailey, A.T., SHaffrey, C.I., Arnold, P.M., Darrel, S., Brodke, D.S., Rampersaud, R., Grauer, J.N., Winegar, C. and Vaccaro, A.R. (2008) Variations in Surgical Treatment of Cervical Facet Dislocations. Spine, 33, E188-E193. https://doi.org/10.1097/BRS.0b013e3181696118

[13] Tofuku, K., Koga, H., Yone, K. and Komiya, S. (2013) Distractive Flexion Injuries of the Subaxial Cervical Spine Treated with a Posterior Procedure Using Cervical Pedicle Screws or a Combined Anterior and Posterior Procedure. Journal of Clinical Neuroscience, 20, 697-701. https://doi.org/10.1016/j.jocn.2012.03.045 
[14] Anderson, P.A., et al. (1991) Posterior Cervical Arthrodesis with AO Reconstruction Plates and Bone Graft. Spine, 16, S72-S79.

https://doi.org/10.1097/00007632-199103001-00012

[15] Nazarian, S.M. and Louis, R.P. (1991) Posterior Internal Fixation with Screw Plates in Traumatic Lesion of the Cervical Spine. Spine, 16, S64-S71. https://doi.org/10.1097/00007632-199103001-00011

[16] Bohlman, H.H. and Anderson, P.A. (1992) Anterior Decompression and Arthrodesis of the Cervical Spine: Long Term Motor Improvement. Part-I-Improvement in Incomplete Traumatic Quadriparesis. The Journal of Bone and Joint Surgery. American Volume, 74A, 671-82.

https://doi.org/10.2106/00004623-199274050-00006

[17] de Oliveria, J.C. (1987) Anterior Plate Fixation of Traumatic Lesions of the Lower Cervical Spine. Spine, 12, 324-329.

https://doi.org/10.1097/00007632-198705000-00003 